\title{
A Retrospective Single Center Study Investigating the Clinical Significance of Grade in Triple Negative Breast Cancer
}

Sarang Upneja ${ }^{1}$, Abdulkadir Hussein ${ }^{2}$, Devinder Moudgil ${ }^{3}$, Swati Kulkarni ${ }^{4}$, Rasna Gupta ${ }^{5}$, Amin Kay ${ }^{6}$, Lisa Porter ${ }^{7}$, Bre-Anne Fifield $^{8}$, Rosa Ferraiuolo ${ }^{8}$ and Caroline $\mathrm{Hamm}^{9 *}$

${ }^{1}$ Schulich School of Medicine and Dentistry, Western University, Windsor, (ON), Canada

${ }^{2}$ Department of Mathematics, University of Windsor, Windsor, (ON), Canada

${ }^{3}$ Department of Oncology, Windsor Regional Hospital, Windsor, (ON), Canada

${ }^{4}$ Schulich School of Medicine and Dentistry, Western University, Windsor, (ON), Canada

${ }^{4}$ Department of Oncology, Windsor Regional Hospital, Windsor, (ON), Canada

${ }^{4}$ Department of Biomedical Science, University of Windsor, Windsor, (ON), Canada

${ }^{5}$ Schulich School of Medicine and Dentistry, Western University, Windsor, (ON), Canada

${ }^{6}$ Schulich School of Medicine and Dentistry, Western University, Windsor, $(\mathrm{ON})$, Canada

${ }^{7}$ Department of Mathematics, University of Windsor, Windsor, (ON), Canada

${ }^{8}$ Department of Biology, University of Windsor, Windsor, (ON), Canada

${ }^{9}$ Schulich School of Medicine and Dentistry, Western University, Windsor, (ON), Canada

${ }^{*}$ Corresponding author: Dr. Caroline Hamm, Schulich School of Medicine and Dentistry, Western University, Windsor, (ON), Canada; Tel: 1-519-890-4382; Email: caroline.hamm@wrh.on.ca

Received: September 29, 2021; Accepted: October 05, 2021; Published: October 08, 2021

\begin{abstract}
The purpose of this study was to investigate the predictive value of histological grade in triple negative breast cancer (TNBC). We retrospectively analyzed 305 TNBC patient charts from 2004-2017 at Windsor Regional Cancer Center with triple negative defined as estrogen (ER), progesterone (PR), and HER-2 negative. The significance of grade with respect to demographic and treatment variables as well as patient outcomes was determined. There were found to be 10,45 , and 250 patients with tumor grades $1,2,3$, respectively. The overall survival rates were $90.12 \%$, $64.4 \%$, and $77.2 \%$, for patients with grade 1, 2 and 3 tumors respectively ( $\mathrm{p}=0.019)$. Overall relapse rates were $70 \%, 55.6 \%$, and $75.6 \%$, respectively for patients with tumor grades 1 , 2 , and 3 ( $p=0.04$ ) Comparing between grade 2 and grade 3, we determined that patients with grade 2 tumors had a 5.5-fold increased risk of death $(\mathrm{HR}=5.513 ; 95 \% \mathrm{CI} 1.2-25.6)$ and shorter time to relapse (HR=1.9; 95\% CI 1.1-3.2) at five years from time of diagnosis. In this retrospective review, grade was shown to have positive predictive value in determining relapse. This finding has the potential to impact patients and their clinicians, and as well, suggests a unique focus on this patient group in future research is recommended.
\end{abstract}

Keywords: Triple negative, Breast cancer, Grade

\section{Introduction}

Triple-negative breast cancer (TNBC) is heterogeneous cancer type, which lacks the receptors for estrogen, progesterone, and human epidermal growth factor receptor-2 proteins [1]. Clinical features associated with TNBC in comparison to other breast cancer subtypes include younger age, less than 50 years, positive breast cancer gene (BRCA 1/2) status, and a family history of breast cancer [2,3].

TNBC cancer sub-type makes up 10-20\% of invasive breast cancer subtypes, and most patients present with significantly larger tumor masses exhibiting rapid growth $[4,5]$. TNBC has an aggressive clinical course, often with lymph node involvement early on at diagnosis, a higher rate of early recurrence, poor short-term prognosis, and increased 5-year risk of mortality [6-8]. The current therapeutic management is primarily chemotherapy and surgical resection of localized tumors $[9,10]$, followed by radiotherapy. Despite advances in novel immunotherapies and the discovery of additional biomarkers serving as therapeutic targets, TNBC remains clinically challenging to treat $[11,12]$. A distant recurrence rate of $33.9 \%$ has been found in TNBC patients compared to a $20.4 \%$ rate amongst patients with non-TNBC [7].

WHO classifies TNBC tumors in histopathological grades from 1 to 3 [13]. Many TNBC disease prognosis and outcome clinical research studies on focused on tumor stage or the extent of cancer spread whereby patients presenting with localized, early-stage cancers have better outcomes than those presenting at a later stage [14]. Tumor size, molecular profiles, and nodal status have also been studied in relation to disease outcome, yet there remains limited information on the 
histological grade's predictive significance in TNBC outcome $[15,16]$. The new American Joint Committee on Cancer (AJCC) Staging System 8 has incorporated grade into the overall prognostic scoring system for breast cancer [17]. On a similar note, a review of the Surveillance, Epidemiology, and End Results (SEER) database found that the tumor grade remains a prognostic factor in breast cancer despite lymph node burden or tumor size [18]. In both systems, grade 3 remains a higher risk for recurrence than grade 2 .

A key marker for defining the biological character of a tumor is the grade or tumor differentiation status. The most widely used grading system is Scarff, Bloom, Richardson, updated by the Nottingham group [19]. This grade for a tumor is determined by assessing morphologic features such as tubule formation, nuclear pleomorphism, and calibrated mitotic count. Grade 3 tumors have the highest unfavorable score [17]. Therefore, of interest are the unique results of non-preplanned subgroup analysis from a recent trial that noted that the smaller group of patients with grade 2 tumors demonstrated a non-statistically significant increase in relapse than patients with grade 3 tumors [20].

Considering this increased recurrence trend and already limited chemotherapy options available to TNBC patients, we must identify TNBC-specific predictive factors influencing patient outcomes. This study investigates the predictive value of grade in TNBC from the Windsor Regional Cancer Program database of 1734 breast cancer patients from 2004-2017.

\section{Materials and Methods}

\section{Study Patients}

Following research ethics board approvals, a previously compiled TNBC database was updated with new patients from 2011-2012 and a cohort of triple negative patients treated with carboplatin as part of Speedy (Spy)1 clinical trials from 2013-2017 [21]. The final database consisted of 305 triple negative breast cancer patients treated at the Windsor Regional Cancer Centre (WRCC) from 2004-2017. Research Ethics Approval was obtained from the Joint REB of Windsor Regional Hospital and University of Windsor \#35666.

\section{Inclusion Criteria}

In this study, triple negative breast cancer was defined as less than or equal to $10 \% \mathrm{ER}, \mathrm{PR}$, HER-2 expression or greater than $10 \%$ for HER-2 considered equivocal and confirmed negative by FISH testing. Tumor grade was recorded in all cases as grade 1, 2, or 3 as listed in patient records or as a grade 3 when reported as 'high grade'.

\section{Exclusion Criteria}

Any patients with a diagnosis prior to 1994 with recurrence after 2004 and/or missing oncological information and patients with cancer types such as Ductal Carcinoma in Situ were excluded due to limited involvement of physiological breast tissue.

\section{Database Variables}

Database variables included the following demographics pertaining to the patient (age, BRCA1/2 status), tumor (ER/PR/HER2 status, size, grade), cancer (pathology type, AJCC-7 stage, lymph node status, both side cancer), and treatments (surgery, radiation, chemotherapy, and hormone therapy).

\section{Outcomes}

The relapse-free and overall survival (OS) was assessed from date of diagnosis to date of last follow-up or death. Relapse free and overall survival data was updated through reviewing newspaper obituaries, Windsor Regional Hospital electronic medical record, and southwest Ontario wide Clinical Connect electronic records as proxy for continued use of health care services.

\section{Statistical Analysis}

Statistical comparisons (t-test or chi square) were carried out among the three grade groups with respect to the relevant demographic and clinical factors. In order to identify pairwise differences between grades, a multiple comparison test was performed. Kaplan-Meier survival analysis was done for survival time and time to relapse but due to a crossing of the curves, a weighted version with higher statistical power, known as Fleming-Harrington test was used instead of the usual Log-Rank Test. Due to the crossing, a COX PH stepwise regression compared overall survival and time to relapse between grades 2 and 3, with time stratified by 5 -year cut point.

\section{Results}

\section{Association between Grade and Clinical Variables}

This study examined a total 305 TNBC cases. $82 \%$ patients were grade $3(n=250), 15 \%$ were grade $2(n=45)$ and $3 \%$ were grade $1(\mathrm{n}=10)$. The median patient age at diagnosis was 56 years however grade 3 tumors were frequently observed in women younger by 3-7 years as compared with grades 1,2 patients $(\mathrm{P}=0.007)$. Results of BRCA testing are available for 50 patients. Seventy per cent of those tested were positive for BRCA1 or BRCA2.

Infiltrating ductal cancer was the predominant histological subtype (91\%). Other histological subtypes were squamous metaplasia, mucinous, papillary, cystic adenoid and atypical medullary. The majority of patients $(87.2 \%)$ had tumor size less than $5 \mathrm{~cm}$ and fewer $(12.8 \%)$ had a tumor size more than $5 \mathrm{~cm}$. There were no statistically significant differences in tumor size between grade 1,2 , and 3 patients. Positive or non-zero ER immunostaining was found in $7.9 \%$ of the cases and PR positivity in $9.5 \%$ cases.

For chemotherapy, $17.4 \%$ of the patients received anthracycline based regimens (Adriamycin/Cyclophosphamide (AC) or 5Florouracil/Epirubicin/Cyclophosphamide (FEC)). Meanwhile, 30\% of patients received an anthracycline/taxane regimen (AC paclitaxel (ACT) or FEC paclitaxel (FECT). Almost thirty per cent $(27.9 \%)$ received carboplatin (ACT+ Carboplatin) and 3.3\% received other chemotherapy types. Eighteen per cent of the patients did not receive any chemotherapy.

The results were statistically insignificant among three tumor grade patient groups for all tested variables except for chemotherapy administration with fewer grade 1 patients receiving chemotherapy $(\mathrm{P}=0.008)$. The small sample size of grade 1 patients $(\mathrm{n}=10)$ and a significant portion of these individuals or $50 \%$ were not treated 
with any chemotherapy which may explain the skewed statistical significance of chemotherapy with grade.

There was also a marginally significantly difference in ER status and hormone therapy distribution $(\mathrm{P}=0.097)$. The majority of patients (92\%) in the study already had a zero \% ER status and 95\% did not receive hormone therapy. A higher percentage of grade 2 patients had some ER positivity, but this did not affect outcomes by multivariate analysis (Table 1).

*The p-value is calculated by ANOVA for numerical covariates and chi-square test for categorical covariates.

\section{The Role of Radiation Therapy in TNBC}

The factors that appeared to be significantly associated with a shorter time to relapse included late stages, chemotherapy and radiation site. The time-independent analysis yielded factors associated with death risk and found that radiation site to a targeted area of breast tissue was deemed statistically preferable than the chest wall.

\section{Comparison of Disease-Free and Overall Survival Times among the Three Tumor Grades}

\section{Effect of Grade on Time to Relapse}

Time from diagnosis of breast cancer to relapse was determined in each patient and analyzed by grade. In this analysis, grade 2 patients had an inferior relapse-free survival than both grade 1 and grade 3 patients. Overall, the relapse-free survival rates were 70, 55.6 and $75.6 \%$, respectively for the three groups (grade 1, 2 and 3 respectively) with a 16-year follow-up. The mean disease-free survival time was 6.8 , 8.7 , and 8.9 years, respectively by grade 1,2 , and 3 . Over the maximal 16-year follow-up, grade 3 patients faired significantly better than grade 2 in terms of disease-free survival $(\mathrm{P}=0.04)$ (Figure 1).

\section{Effect of Grade on Overall Survival}

Time from diagnosis to death or last follow-up was analyzed from all 305 patients to determine overall survival (OS) times for the patient subgroups stratified by grade 1, 2 and 3 tumors. Among the 3 grades, grade 2 patients had poorest OS at $64.4 \%$ while survival rates for grade 1 and grade 3 were $90.12 \%$ and $77.2 \%$ ( $\mathrm{p}=0.019$ ), respectively at 5 years.

We observed that grade 2 patients had better OS during the first three years of treatment followed by worse OS than the other 2 groups in the following years, with a maximum follow-up time of 16 years. This is apparent from the Kaplan-Meyer curves for grades 2 and 3 crossing each other at about three years from date of diagnosis (Figure 2). Due to this crossing, the usual log-rank test suffered from low statistical power and therefore we employed a weighted version, known as Fleming-Harrington test. This method placed more emphases on the differences in survival between the groups after the

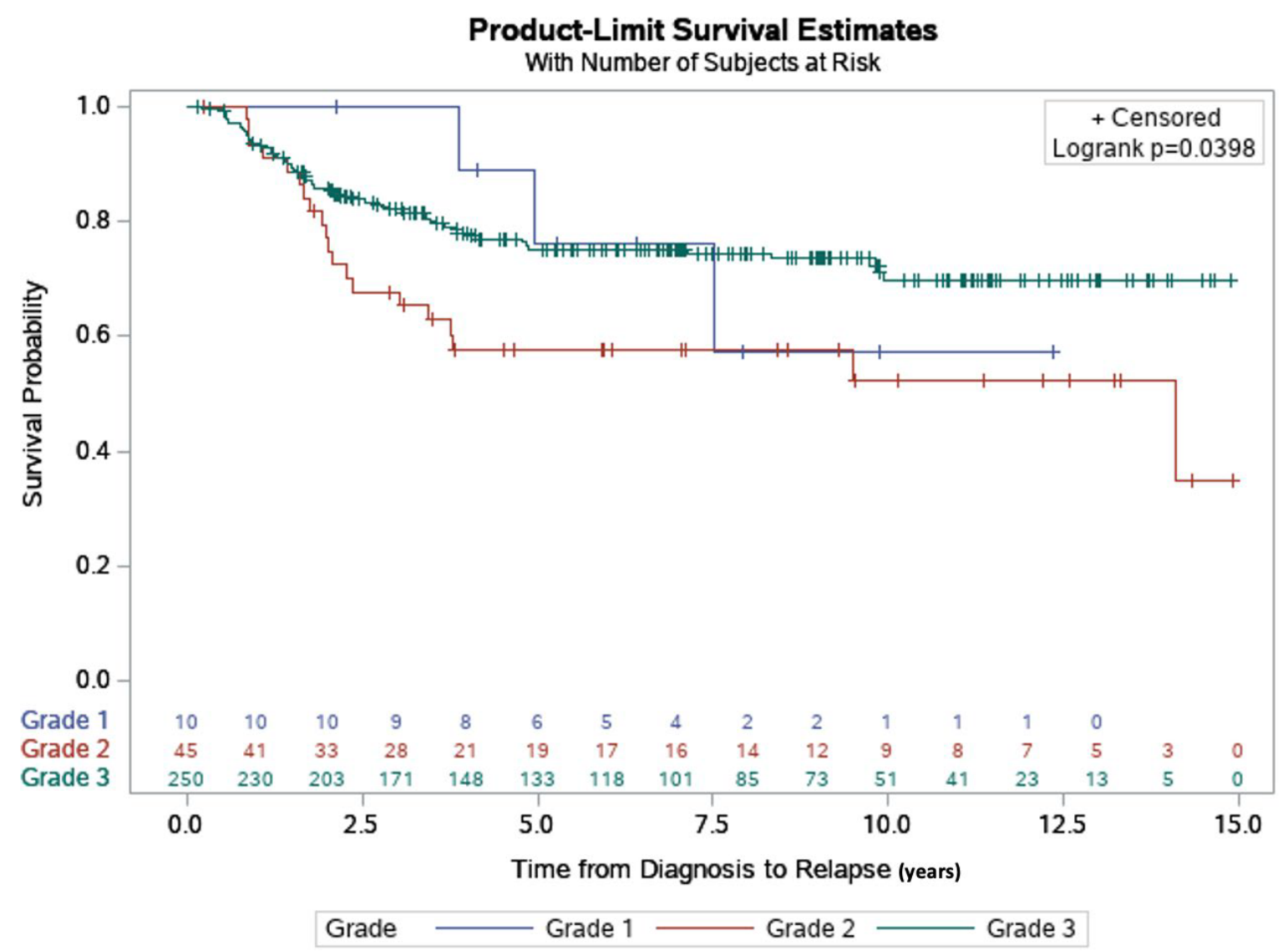

Figure 1: Relapse-free survival estimate by grade. The relapse-free rate by grade, of 305 TNBC patients that were followed for a 16-year maximal follow-up time. Time to relapse (years) was depicted using Kaplan-Meier curves. Overall, the relapse-free survival rates were 70, 55.6 and $75.6 \%$, respectively for the three groups. The mean relapse-free survival times for the three groups were, $6.8,8.7$ and 8.9 years, respectively for Grades 1,2 and $3(\mathrm{p}=0.04)$. 
Table 1: Analysis of Grade with Clinical Variables.

\begin{tabular}{|c|c|c|c|c|c|}
\hline \multirow[b]{2}{*}{ Variable } & \multicolumn{5}{|c|}{ Grade } \\
\hline & Grade $1(N=10)$ & Grade $2(N=45)$ & Grade $3(\mathrm{~N}=250)$ & Total $(\mathrm{N}=305)$ & p value \\
\hline Age & & & & & 0.007 \\
\hline Mean (SD) & $58.400(12.580)$ & $61.067(12.646)$ & $54.512(13.196)$ & $55.607(13.271)$ & \\
\hline Range & $43-78$ & $37-86$ & $25-89$ & $25-89$ & \\
\hline Stage (AJCC-7) & & & & & 0.498 \\
\hline IA & $3(30.0 \%)$ & $17(37.8 \%)$ & $60(24.0 \%)$ & $80(26.2 \%)$ & \\
\hline IIA,B & $6(60.0 \%)$ & $19(42.2 \%)$ & $136(54.4 \%)$ & $161(52.8 \%)$ & \\
\hline IIIA,B,C & $1(10.0 \%)$ & $9(20.0 \%)$ & $50(20.0 \%)$ & $60(19.7 \%)$ & \\
\hline IV & $0(0.0 \%)$ & $0(0.0 \%)$ & $4(1.6 \%)$ & $4(1.3 \%)$ & \\
\hline Tumor Size $(\mathrm{cm})$ & & & & & 0.487 \\
\hline $0-1.9$ & $4(40.0 \%)$ & $18(40.0 \%)$ & $73(29.2 \%)$ & $95(31.1 \%)$ & \\
\hline $2-4.9$ & $5(50.0 \%)$ & $20(44.4 \%)$ & $146(58.4 \%)$ & $171(56.1 \%)$ & \\
\hline$>5$ & $1(10.0 \%)$ & $7(15.6 \%)$ & $31(12.4 \%)$ & $39(12.8 \%)$ & \\
\hline Chemotherapy & & & & & 0.008 \\
\hline $\mathrm{AC} / \mathrm{CEF} / \mathrm{FEC}$ & $1(10.0 \%)$ & $7(15.6 \%)$ & $45(18.0 \%)$ & $53(17.4 \%)$ & \\
\hline ACT/FECT & $4(40.0 \%)$ & $14(31.1 \%)$ & $84(33.6 \%)$ & $102(33.4 \%)$ & \\
\hline ACT + Carbo & $0(0.0 \%)$ & $7(15.6 \%)$ & $78(31.2 \%)$ & $85(27.9 \%)$ & \\
\hline Other (TC, CMF, FEC+FUC) & $0(0.0 \%)$ & $3(6.7 \%)$ & $7(2.8 \%)$ & $10(3.3 \%)$ & \\
\hline None & $5(50.0 \%)$ & $14(31.1 \%)$ & $36(14.4 \%)$ & $55(18.0 \%)$ & \\
\hline ER Status & & & & & 0.086 \\
\hline $0 \%$ & $10(100.0 \%)$ & $38(84.4 \%)$ & $233(93.2 \%)$ & $281(92.1 \%)$ & \\
\hline Rest & $0(0.0 \%)$ & $7(15.6 \%)$ & $17(6.8 \%)$ & $24(7.9 \%)$ & \\
\hline PR Status & & & & & 0.565 \\
\hline $0 \%$ & $10(100.0 \%)$ & $41(91.1 \%)$ & $225(90.0 \%)$ & $276(90.5 \%)$ & \\
\hline Rest & $0(0.0 \%)$ & $4(8.9 \%)$ & $25(10.0 \%)$ & $29(9.5 \%)$ & \\
\hline Pathology Type & & & & & 0.833 \\
\hline Infiltrating & $9(90.0 \%)$ & $40(88.9 \%)$ & $229(91.6 \%)$ & $278(91.1 \%)$ & \\
\hline $\begin{array}{l}\text { Other (squamous metaplasia, mucinous, papillary, } \\
\text { cystic adenoid, atypical medullary) }\end{array}$ & $1(10.0 \%)$ & $5(11.1 \%)$ & $21(8.4 \%)$ & $27(8.9 \%)$ & \\
\hline BRCA 1/2 Status & & & & & 0.734 \\
\hline Negative & $1(10.0 \%)$ & $3(6.7 \%)$ & $31(12.4 \%)$ & $35(11.5 \%)$ & \\
\hline Positive & $0(0.0 \%)$ & $3(6.7 \%)$ & $12(4.8 \%)$ & $15(4.9 \%)$ & \\
\hline N/A (Not Tested) & $9(90.0 \%)$ & $39(86.7 \%)$ & $207(82.8 \%)$ & $255(83.6 \%)$ & \\
\hline Radiation Site & & & & & 0.681 \\
\hline None & $2(20.0 \%)$ & $17(37.8 \%)$ & $70(28.0 \%)$ & $89(29.2 \%)$ & \\
\hline Breast & $6(60.0 \%)$ & $21(46.7 \%)$ & $130(52.0 \%)$ & $157(51.5 \%)$ & \\
\hline Chest Wall & $2(20.0 \%)$ & $7(15.6 \%)$ & $50(20.0 \%)$ & $59(19.3 \%)$ & \\
\hline Surgery Type & & & & & 0.926 \\
\hline None & $0(0.0 \%)$ & $0(0.0 \%)$ & $1(0.4 \%)$ & $1(0.3 \%)$ & \\
\hline Mastectomy (M) & $0(0.0 \%)$ & $3(6.7 \%)$ & $21(8.4 \%)$ & $24(7.9 \%)$ & \\
\hline M + Axillary LN & $3(30.0 \%)$ & $14(31.1 \%)$ & $74(29.7 \%)$ & $91(29.9 \%)$ & \\
\hline M + Sentinel LN & $1(10.0 \%)$ & $2(4.4 \%)$ & $17(6.8 \%)$ & $20(6.6 \%)$ & \\
\hline Lumpectomy (L) & $0(0.0 \%)$ & $1(2.2 \%)$ & $4(1.6 \%)$ & $5(1.6 \%)$ & \\
\hline $\mathrm{L}+$ Axillary LN & $3(30.0 \%)$ & $15(33.3 \%)$ & $62(24.9 \%)$ & $80(26.3 \%)$ & \\
\hline L + Sentinel LN & $3(30.0 \%)$ & $6(13.3 \%)$ & $59(23.7 \%)$ & $68(22.4 \%)$ & \\
\hline Other (Multiple) & $0(0.0 \%)$ & $4(8.9 \%)$ & $11(4.4 \%)$ & $15(4.9 \%)$ & \\
\hline N/A & 0 & 0 & 1 & 1 & \\
\hline Hormone Therapy & & & & & 0.097 \\
\hline None & $10(100.0 \%)$ & $40(88.9 \%)$ & $240(96.0 \%)$ & $290(95.1 \%)$ & \\
\hline Received & $0(0.0 \%)$ & $5(11.1 \%)$ & $10(4.0 \%)$ & $15(4.9 \%)$ & \\
\hline Number of LN Positive & & & & & 0.543 \\
\hline Mean (SD) & $2.200(4.392)$ & $2.089(4.502)$ & $1.484(3.647)$ & $1.597(3.802)$ & \\
\hline Range & $0.000-13.000$ & $0.000-21.000$ & $0.000-24.000$ & $0.000-24.000$ & \\
\hline
\end{tabular}




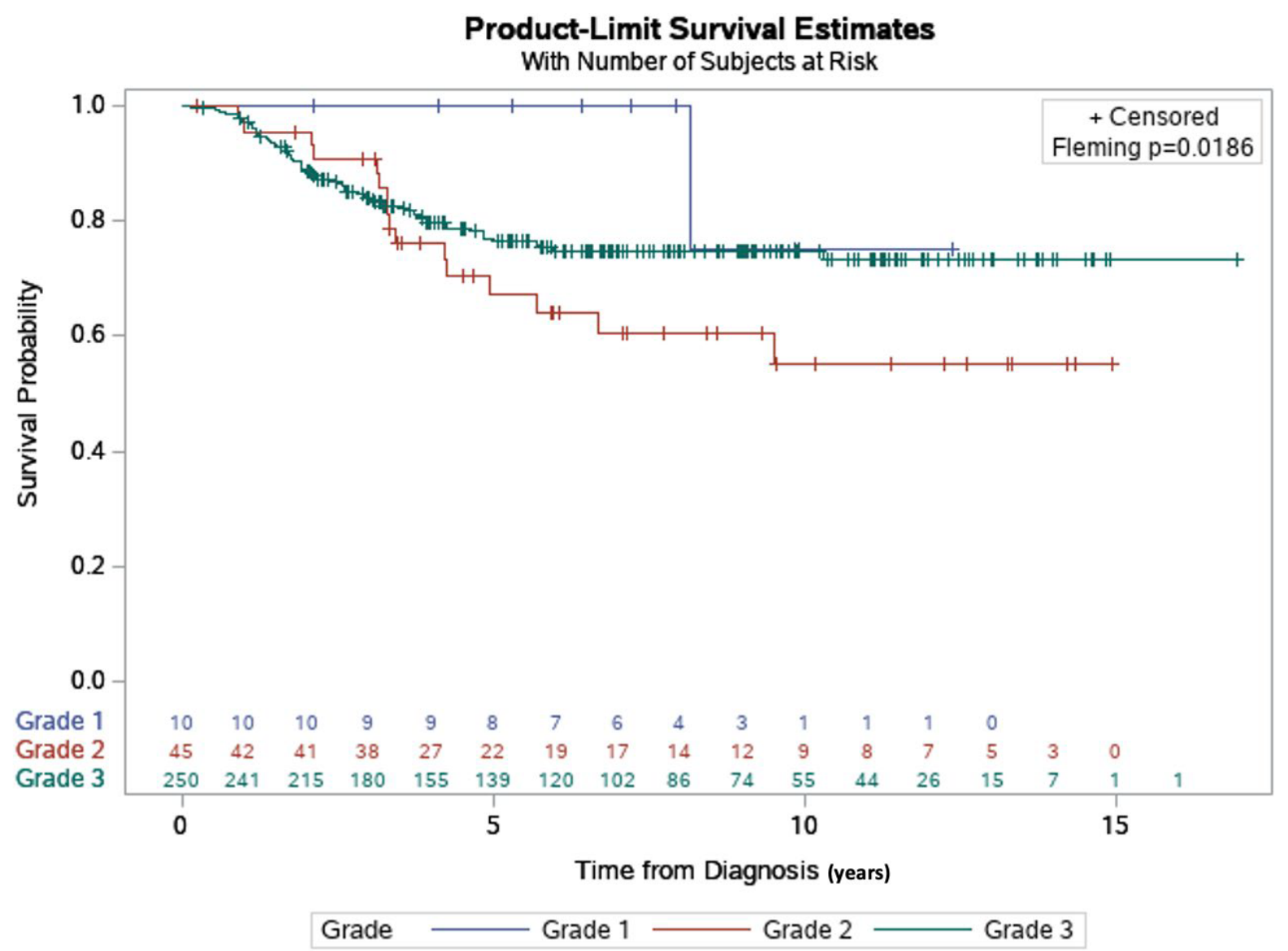

Figure 2: Survival estimate by grade and time of diagnosis. The OS rates, by grade, of 305 TNBC patients that were followed for a 16-year maximal follow-up time. Survival (years) was depicted using Kaplan-Meier survival estimates. Overall, the survival rates were $90.12 \%$ and $64.4 \%$ and $77.2 \%$, respectively for Grades 1,2 , and 3 ( $\mathrm{p}=0.019$ ).

curves crossed which was 3 years from the time of the diagnosis to maximum follow-up.

The overall results showed that grade 2 patients had a 5.9-fold increased risk of death after the first five years from diagnosis, while before five years, the difference was not statistically significant $(\mathrm{HR}=5.930 ; 95 \%$ CI 1.2-27.3). Additionally, grade 2 patients were shown to have a 2 -fold sooner time to relapse $(\mathrm{HR}=1.888,95 \% \mathrm{CI}$ 1.1-3.2).

The OS rates, by grade, of 305 TNBC patients that were followed for a 16 year maximal follow-up time. Survival (years) was depicted using Kaplan-Meier survival estimates and Fleming-Harrington weighted testing due to the curves' crossing. A statistically significant difference was then found among the three groups in terms of overall survival by grade $(\mathrm{P}=0.019)$. We followed this up with a statistical multiple comparison test to determine any pairwise differences among the three groups. The analysis found a statistically significant difference between grades 1 and 2, 2 and 3, but not 1 and 3 .

\section{Statistical Considerations Regarding the Survival Analyses}

To elucidate the significance of grade 2 patients standing out from other grades during the pairwise testing, we stratified these cohorts of patients by time using five years as a cut point. As can be seen from the crossing of the Kaplan-Meyer curves (Figures 1 and 2), one can infer that after about 3 years from time of diagnosis, grade 2 patients did worse than grade 3 . A conservative assumption is that the curves depart from each other after 5 years. In this way, the stratification helped detect a significant grade effect on survival after the initial five years. A limitation of this approach is ad hoc time cut off at 5 years. There are no established statistical methods to better determine the time cut off.

It is important to note here that attention was restricted to comparing the differences between grade 2 and grade 3 only. The small sample size of grade 1 as well as lack of pairwise statistical significance between grade 1 and 2, did not lend itself in further delineating and uncovering the significance of grade in this study.

We did a model selection using stepwise regression and found that the factors that appeared to be significantly associated with the risk of death-independent of the time interval - were stage, the chemotherapy, and radiation site (Table 1). Specifically, patients with late stage cancers (IV), those who did not receive chemotherapy, and ones who received radiation to the chest wall as opposed to the breast, had worse outcomes. As to the outcomes associated with specific chemotherapy subtype such as ACT vs. Carboplatin, the results were not found to be statistically significant at this time. 


\section{Discussion}

TNBC can be divided into six different subtypes by microarray [22,23]. The pivotal report by Perou re-defined TNBC into five subtypes and illustrated significant heterogeneity of TNBC. Research to identify more readily available prognostic characteristics in this patient group is ongoing since micro-array analyses are not available in the majority of clinical settings [24-26]. Recent reports have identified different low-grade histologies that are triple-negative $[27,28]$ In our study, $89 \%$ of those in our study were invasive ductal carcinomas. Kwon et al demonstrated that the modified Nottingham Prognostic Index, which includes tumor size, nodal status, and grade as the most critical factor in determining outcome in a patient with TNBC [29]. Chollet et al. also demonstrated this importance of grade showing that grade was the only significant factor for survival in patients treated in the neoadjuvant fashion [30].

The importance of grade in ER-positive tumors has been examined previously [31-33]. A recommendation from the 2009 St. Gallen International Expert Consensus on the 'Primary Therapy of Early Breast Cancer' suggested that grade 1 and grade 3 be taken into consideration to assess indications of adjuvant chemotherapy in ERpositive patients [31]. Grade 2 was regarded similar to other parameters of intermediate-risk significance: however, there was no comment on the importance of grade in the TNBC patient population. Two retrospective database reviews from SEER underlined the importance of grade in breast cancer patients' outcomes. However, neither study examined the TNBC patient population as a separate sub-group and TNBC patients were included in the analysis without stratification $[18,32]$. Another study presented at the St. Gallen's meeting examined ER-positive patients but only in determining the importance of grade in breast cancer patient outcomes [34].

In our study, $82 \%$ of TNBC patients had grade 3 tumors, and $18 \%$ had grade 1 and 2 tumors. This is similar to the findings of other reports in which grade 3 patients make up the majority of patient set $[26,29,35]$. However, here we present an important and unique feature of grade in TNBC with the surprising finding that patients with grade 2 tumors have worse long-term outcomes compared with grade 3 tumors.

Interestingly, we found that patients with grade 2 tumors experienced better progression-free and overall survival for the first three-five years, followed by a significant decline in PFS and OS and a 5.9-fold increased risk of death compared with grade 3 tumor patients. It was interesting to note that the grade 2 patients had pairwise, significant differences with both counterparts: grade 1 and 3; but the grades 1 and 3 did not have any significant differences with each other. There may be unknown genomic and molecular mechanisms at play between high and low-grade lesions thus necessitating a closer look into histological subtyping, molecular subtyping and cell biology of TNBC tumors [24,36,37].

The definition of ER/PR negativity at $<10 \%$ in this study is a weakness. Although this was a historically accepted, newer guidelines restrict HR negativity to $0 \%$ [38]. The marginal significance of ER status and hormone therapy can be explained; only $8 \%$ of patients had 1-9\% ER positivity, and 9.5\% had PR positivity. Only 5\% received any hormone therapy. In grade 2 patients, $15.6 \%$ had $1-9 \%$ ER positivity versus $6.8 \%$ in the grade 3 patient population. In the grade 2 population, $8.8 \%$ had $0-9 \%$ PR positivity and $10 \%$ of the grade 3 had $0-9 \mathrm{PR}$ positivity. Although a higher percentage of patients with grade 2 tumors had $1-9 \%$ HR positivity, this did not predict for relapse in this patient population.

A closer look into TNBC patient population is necessary in examining the importance of grade in patient. Curiously, the grade 2 patients fared better for the first three years, but then lose this advantage. Molecular profiling, such as utilizing miRNA changes and examination of the tumor at the time of relapse, may help determine the reasons behind this relapse pattern as the grade 2 tumors may change more often than the grade 3 tumors [39-41].

The increased number of $\mathrm{HR}+$ patients in this group (27 out of 305) also supports the likelihood of a unique molecular profile in this patient population. Additional studies are underway to investigate this finding while ongoing research is focusing on changes in HR/ HER-2 expression at relapse in TNBC with implications in adjusting chemotherapy options for better patient outcomes [42]. Subsequent molecular profiling at relapse can also determine differential expression of biomarkers such as programmed cell death ligand (PD-1/PD-L1) or cell proliferation protein speedy (Spy1) [43], by grade [44]. This may open up novel targets for triple-negative breast cancer treatment by introducing Cyclin inhibitors' potential in future neoadjuvant clinical trials [45].

This study used the AJCC 7 staging however, the new 2018 AJCC 8 staging guidelines have incorporated grade into the staging system of a breast cancer patient. In our study, a T2N0M0 grade 3 breast cancer patient is staged as IIA; however, with AJCC 8, the system would upstage this patient to IIB. Our findings question that grade 3 might be favorable and downstage the patient, whereas a grade 2 tumor may upstage the patient.

Although there is increasing information about low-grade TNBC, this study offers clinical outcomes for those diagnosed with grade 2 invasive ductal carcinoma. This report has all of the limitations of a retrospective study but has intriguing findings of worse outcomes in grade 2 TNBC. Incorporating grade into the stratification of analysis for future TNBC cases would clarify the importance of grade in TNBC overall survival.

Limitations of this analysis include the retrospective nature of the analysis. As well, the inclusion of the $1-9 \%$ HR positivity in the analysis may have influenced the outcomes of our analysis, and stricter criteria of HR positivity may influence these results.

\section{Conclusion}

In this retrospective review, we found that Grade 2 in TNBC was shown to have a negative prognostic value in determining progression-free survival and overall survival. This is paradoxical to non-TNBC where grade 3 has worse long-term outcomes than grade 2. Long term follow-up of TNBC patients is necessary to elucidate this phenomenon as we noted a difference in outcome in shorter follow 
up versus longer follow-up. TNBC patients with grade 2 tumors experienced inferior disease-free survival and overall survival with long term follow-up, with a six-fold increased risk of death. We are currently planning collaborative research using a stricter definition of ER PR status to expand this data set to further investigate the issue of grade in triple negative breast cancer. If this finding is confirmed, it would have significant and easily translatable prognostic information for patients and clinicians alike in the triple negative breast cancer population.

\section{Funding}

This research received funding from the South Western Ontario Research Program, Western University, and the University of Windsor.

\section{Institutional Review Board Statement}

Research Ethics Approval was obtained from the Joint REB of Windsor Regional Hospital and University of Windsor \#35666.The study was conducted according to the guidelines of the Declaration of Helsinki, and approved by the Institutional Review Board (or Ethics Committee) of Windsor Regional Hospital and the University of Windsor (protocol code 35666, Feb 27, 2019)

\section{Informed Consent Statement}

Patient consent was waived, per institutional guidelines. No patient identifiable data was used in this research. All patient information was de-identified prior to analysis and report development.

\section{Data Availability Statement}

Data available on request due to restrictions in privacy or ethical reasons. The data presented in this study are available on request from the corresponding author. The data are not publicly available as this data set is based on hospital-based data.

\section{Acknowledgments}

We acknowledge Windsor Regional Hospital for their support in facilitating this research.

\section{References}

1. Foulkes WD, Smith IE, Reis-Filho JS (2010) Triple-negative breast cancer. N Engl J Med 363: 1938-1948

2. Gierach GL, Burke A, Anderson WF (2010) Epidemiology of triple negative breast cancers. Breast Dis 32: 5-24. [crossref]

3. Yeh J, Chun J, Schwartz S, Wang A, Kern E, et al. (2017) Clinical Characteristics in Patients with Triple Negative Breast Cancer. International Journal of Breast Cancer 2017: 1796145. [crossref]

4. Boyle P (2012) Triple-negative breast cancer: epidemiological considerations and recommendations. Annals of Oncology 23: 7-12. [crossref]

5. Plasilova ML, Hayse B, Killelea BK, Horowitz NR, Chagpar AB, et al. (2016) Features of triple-negative breast cancer: Analysis of 38,813 cases from the national cancer database. Medicine (Baltimore) 95: 4614. [crossref]

6. Dawson SJ, Provenzano E, Caldas C (2009) Triple negative breast cancers: clinical and prognostic implications. Eur J Cancer 45: 27-40. [crossref]

7. Dent R, Trudeau M, Pritchard KI, Hanna WM, Kahn HK, et al. (2007) TripleNegative Breast Cancer: Clinical Features and Patterns of Recurrence. Clinical Cancer Research 13: 4429-4434. [crossref]

8. Lin NU, Vanderplas A, Hughes ME, Theriault RL, Edge SB, et al. (2012)
Clinicopathologic features, patterns of recurrence, and survival among women with triple-negative breast cancer in the National Comprehensive Cancer Network. Cancer 118: 5463-5472. [crossref]

9. Li CH, Karantza V, Aktan G, Lala M (2019) Current treatment landscape for patients with locally recurrent inoperable or metastatic triple-negative breast cancer: a systematic literature review. Breast Cancer Research 21: 143. [crossref]

10. Schmadeka R, Harmon BE, Singh M (2014) Triple-Negative Breast Carcinoma: Current and Emerging Concepts. American Journal of Clinical Pathology 141: 462477. [crossref]

11. Bianchini G, Balko JM, Mayer IA, Sanders ME, Gianni L (2016) Triple-negative breast cancer: challenges and opportunities of a heterogeneous disease. Nat Rev Clin Oncol 13: 674-690. [crossref]

12. Park JH, Ahn JH, Kim SB (2018) How shall we treat early triple-negative breast cancer (TNBC): from the current standard to upcoming immuno-molecular strategies. ESMO Open 3: 000357.

13. World Health Organization. Regional Office for the Eastern, M. Guidelines for management of breast cancer. 2006

14. Hamm C, Kulkarni S, Gupta R, Kay A, Mathews J, et al. (2016) Early Stage Triple Negative Breast Cancer Has Significantly Better Outcomes than More Advanced Disease: A Single Centre Retrospective Review. Journal of Cancer Therapy 7: 665-674.

15. Elnashar AT, Ali ESM, Gaber A (2012) The prognostic value of triple negative in stage II/III breast cancer. Journal of Oncology Pharmacy Practice 18: 68-75. [crossref]

16. Ovcaricek T, Frkovic SG, Matos E, Mozina B, Borstnar S (2011) Triple negative breast cancer -prognostic factors and survival. Radiol Oncol 45: 46-52. [crossref]

17. Badve S, Beitsch P, Bose S, Byrd D, Chen V, et al. (2018) Cancer Staging Manual Eighth Edition.

18. Schwartz AM, Henson DE, Chen D, Rajamarthandan S (2014) Histologic grade remains a prognostic factor for breast cancer regardless of the number of positive lymph nodes and tumor size: a study of 161708 cases of breast cancer from the SEER Program. Arch Pathol Lab Med 138: 1048-1052. [crossref]

19. Elston C, Ellis I, Elston CW, Ellis IO (2002) The value of histological grade in breast cancer: experience from a large study with long term follow up. Histopathology 19: 403-410.

20. Hamm C. A Prospective Adjuvant Trial of Carboplatin based treatment in Triple Negative Breast Cancer.

21. Trials, C.C. Spy 1.

22. Perou CM, Sorlie T, Eisen MB, van de Rijn M, Jeffrey SS, et al. (2000) Molecular portraits of human breast tumours. Nature 406: 747-752. [crossref]

23. Santonja A, Sánchez-Muñoz A, Lluch A, Chica-Parrado MR, Albanell J, et al. (2018) Triple negative breast cancer subtypes and pathologic complete response rate to neoadjuvant chemotherapy. Oncotarget 9: 26406-26416. [crossref]

24. Penault-Llorca F, Viale G (2012) Pathological and molecular diagnosis of triplenegative breast cancer: a clinical perspective. Ann Oncol 23: 19-22. [crossref]

25. Eden P, Ritz C, Rose C, Ferno M, Peterson C (2004) “Good Old” clinical markers have similar power in breast cancer prognosis as microarray gene expression profilers. Eur J Cancer 40: 1837-1841. [crossref]

26. Alsaleem MA, Ball G, Toss MS, Raafat S, Aleskandarany M, et al. (2020) A novel prognostic two-gene signature for triple negative breast cancer. Mod Pathol 33: 22082220

27. Ginter P, Zhiyan Fu, Shin S (2020) Low-grade variants of triple negative breast carcinoma: a review. Diagnostic Histopatholgy 26: 51-60.

28. Geyer FC, Pareja F, Weigelt B, Rakha E, Ellis IO, et al. (2017) The Spectrum of TripleNegative Breast Disease: High-and Low-Grade Lesions. Am J Pathol 187: 2139-2151. [crossref]

29. Kwon J, Eom KY, Koo TR, Kim BH, Kang E, et al. (2017) A Prognostic Model for Patients with Triple-Negative Breast Cancer: Importance of the Modified Nottingham Prognostic Index and Age. J Breast Cancer 20: 65-73. [crossref]

30. Chollet P, Amat S, Belembaogo E, Cure H, de Latour M, et al. (2003) Is Nottingham prognostic index useful after induction chemotherapy in operable breast cancer? $\mathrm{Br} \mathrm{J}$ Cancer 89: 1185-1191. [crossref] 
31. Goldhirsch A, Ingle JN, Gelber RD, Coates AS, Thürlimann B, et al. (2009) Thresholds for therapies: highlights of the St Gallen International Expert Consensus on the primary therapy of early breast cancer 2009. Ann Oncol 20: 13191329. [crossref]

32. Henson DE, Ries L, Freedman LS, Carriaga M (1991) Relationship among outcome, stage of disease, and histologic grade for 22,616 cases of breast cancer. The basis for a prognostic index. Cancer 68: 2142-2149. [crossref]

33. Rakha EA, Reis-Filho JS, Baehner F, Dabbs DJ, Decker T, et al. (2010) Breast cancer prognostic classification in the molecular era: the role of histological grade. Breast Cancer Research 12: 20. [crossref]

34. Ehinger A, Malmström P, Bendahl PO, Elston CW, Falck AK, et al. (2017) Histological grade provides significant prognostic information in addition to breast cancer subtypes defined according to St Gallen 2013. Acta Oncol 56: 68-74. [crossref]

35. Abdul Aziz AA, Md Salleh MS, Ankathil R (2020) Clinicopathological and Prognostic Characteristics of Malaysian Triple Negative Breast Cancer Patients Undergoing TAC Chemotherapy Regimen. Int J Breast Cancer 2020: 8424365.

36. Geyer FC, Pareja F, Weigelt B, Rakha E, Ellis IO, et al. (2017) The Spectrum of TripleNegative Breast Disease: High-and Low-Grade Lesions. The American Journal of Pathology 187: 2139-2151. [crossref]

37. Jung K, Gupta N, Wang P, Lewis JT, Gopal K, et al. (2015) Triple negative breast cancers comprise a highly tumorigenic cell subpopulation detectable by its high responsiveness to a Sox2 regulatory region 2 (SRR2) reporter. Oncotarget 6: 1036610373. [crossref]
38. Hammond ME, Hayes DF, Dowsett M, Allred DC, Hagerty KL, et al. (2010) American Society of Clinical Oncology/College Of American Pathologists guideline recommendations for immunohistochemical testing of estrogen and progesterone receptors in breast cancer. J Clin Oncol 28: 2784-2795.

39. Fu F, Xiao X, Zhang T, Zou Q, Chen Z, et al. (2016) Expression of receptor protein tyrosine phosphatase [zeta] is a risk factor for triple negative breast cancer relapse. Biomedical Reports 4: 167-172.

40. Kleivi Sahlberg K, Bottai G, Naume B, Burwinkel B, Calin GA, et al. (2015) Serum MicroRNA Signature Predicts Tumor Relapse and Survival in Triple-Negative Breast Cancer Patients. Clinical Cancer Research 21: 1207-1214. [crossref]

41. Nishimura R, Osako T, Okumura Y, Tashima R, Toyozumi Y, et al. (2011) Changes in the ER, PgR, HER2, p53 and Ki-67 biological markers between primary and recurrent breast cancer: discordance rates and prognosis. World J Surg Oncol 9: 131.

42. Porter, L. 2019.

43. Ferraiuolo RM, Tubman J, Sinha I, Hamm C, Porter LA (2017) The cyclin-like protein, SPY1, regulates the ERalpha and ERK1/2 pathways promoting tamoxifen resistance. Oncotarget 8: 23337-23352. [crossref]

44. Ferraiuolo RM, Tubman J, Sinha I, Hamm C, Porter LA (2017) The cyclin-like protein, SPY1, regulates the ERa and ERK1/2 pathways promoting tamoxifen resistance. Oncotarget 8: 23337-23352. [crossref]

45. Garrido-Castro AC, Lin NU, Polyak K (2019) Insights into Molecular Classifications of Triple-Negative Breast Cancer: Improving Patient Selection for Treatment. Cancer Discovery 9: 176

\section{Citation:}

Upneja S, Hussein A, Moudgil D, Kulkarni S, Gupta R, et al. (2021) A Retrospective Single Center Study Investigating the Clinical Significance of Grade in Triple Negative Breast Cancer. Cancer Stud Ther J Volume 6(3): 1-8. 